\title{
Bernhard Meuser/
}

Schönberger

\section{Bücher machen nach} Sinus-Milieus

Eine von der Deutschen Bischofskonferenz in Auftrag gegebene soziodemografische Untersuchung hat Aufsehen erregt. Es ging um die religiösen und kirchlichen Orientierungen in den Sinus-Milieus 2005. Aus den Ergebnissen dieser Untersuchung entstand ein ZielgruppenHandbuch. In dem wurde nicht nur der soziokulturelle Wandel in Deutschland seit den 50er Jahren bis heute beschrieben, sondern konkrete Antworten auf die drängende Frage gegeben: Wie kann die katholische Kirche die Menschen heute im 21. Jahrhundert in ihren unterschiedlichen Lebenswelten erreichen?

Warum macht Kirche das? Die katholische Kirche hatte festgestellt, dass ein vielleicht noch in den 60er Jahren trennungsscharf aus der Nachkriegsgesellschaft hervorstechendes "katholisches Milieu“ verschwunden war. Dass sich einerseits Konventionen und Gewohnheiten der Menschen immer schneller ändern, differenzieren und individualisieren und dass Religion und Kirche eine zunehmend geringere Rolle im Leben der Menschen spielen. Der Kommunikationsfaden zu einem großen und wachsenden Teil der Bevölkerung droht abzureißen oder ist bereits abgerissen. Eine Entwicklung, der man nicht tatenlos zuschauen kann. Denn eine Kirche, die der Welt die universelle Heilsbotschaft Jesu Christi anzubieten hat, kann sich nicht zum elitären Club entwickeln und in leere Kathedralen zurückziehen. Um den Faden zur Gesellschaft wieder anzuknüpfen braucht es eine Erfolg versprechende Kommunikationsstrategie und ein stimmiges und konkretes Angebot. Das aber setzt eine umfassende Zuwendung zum Menschen voraus. Wie soll die funktionieren, wenn Kirche immer weniger versteht, wie Menschen heute „ticken“?

\section{Warum man heute Sociovision und Marketing braucht}

Wenn die Menschen mit den Füßen abstimmen und der Gesprächsfaden gerissen ist, können manchmal Profis für Kommunikation und Marketing helfen. Das machen Wirtschaft und Politik auch so. Politiker helfen sich mit den Instrumenten der Demoskopie. Unternehmen aus der Konsumgüterindustrie und dem Handel wenden sich häufig an das renommierte sozialwissenschaftliche Institut Sinus Sociovision in Heidelberg. Die haben besondere Erfahrung mit der Definition von Zielgruppen. Dort hat man über Jahre ein dynamisches Beschreibungsmodell der Nachkriegsgesellschaft und ihre Ausdifferenzierung in den sogegannten Sinus-Mi- 
lieus entwickelt. Der Vorteil dieses Gesellschaftsmodells gegenüber konkurrierenden Modellen, die sich mit harten soziodemografischen Daten wie Alter, Geschlecht, Beruf, Einkommen und Bildungsabschluss begnügen, liegt darin, dass es weiche Faktoren wie Konsumgewohnheiten, Mediennutzung, Vorlieben, Ästhetik und Orientierungen zur komplexen Beschreibung von für verschiedene Milieus typische Lebenswelten und Lebensstile verdichtet. Aus diesen Lebensweltbeschreibungen lassen sich qualifiziertere Aussagen hinsichtlich des Konsumverhaltens, der Kommunikationsgewohnheiten und insbesondere der Mediennutzung der Zielgruppen gewinnen. Für Wirtschaft und Handel - und Verlage und Buchhandel gehören hier dazu - ist es entscheidend zu wissen, wie sich diese Lebenswelten sogar bei scheinbar identischen soziodemografischen Zwillingsbiografien unterscheiden. Da kann es beispielsweise den 42 jährigen Bauingenieur geben, der ohne Bücher in Gelsenkirchener Barock lebt und oben drüber sein gleichaltriger Berufskollege in der identisch geschnittenen Wohnung, der sich in Bauhausklassikern und seiner Bibliothek eingerichtet hat. Offensichtlich liegen hier völlig unterschiedliche Milieus und Konsumverhalten vor.

Für Büchermacher und Buchhandel sind deshalb Sinus-Milieus schon länger keine Fremdworte mehr. Wer Bücher macht, die Leser finden und die gekauft werden sollen, muss sein Angebot auf die Bedürfnisse, die Seh- und Lesegewohnheiten, auf Erwartungen und ästhetische Vorlieben seiner potentiellen Kunden abstimmen. Zugegebenermaßen gilt das mehr für den kommerziell ausgerichteten Publikumsverlag, der sich allein aus dem Verkauf seiner Bücher refinanziert und nicht auf kirchliche Subventionen zurückgreifen kann. Publikumsverleger wissen, welche Milieus besonders „buchaffin" sind und welche nie Bücher kaufen. Aber mit dem Büchermachen allein ist es nicht getan, auch das Marketing muss stimmen. Man muss wissen, wie die Zielgruppen, die Milieus, „ticken“ und wie man mit ihnen kommunizieren kann. Wie arbeiten heute Buchverlage mit Sinus-Milieus?

\section{Der Verlagsalltag mit Sinus}

Zunächst muss man sich in Erinnerung rufen, dass jede Kreation eines Buches ein hochkomplexer Vorgang im Rahmen eines gedachten Kommunikationsvorganges ist. Was im großen Stil betrieben wird, wenn ein Automobilproduzent ein neues Auto designt, das geschieht im Kleinen jedes Mal nichf anders, wenn ein Verlag aus einer Idee ein Buch macht, das natürlich möglichst viele Menschen erreichen soll. Wie das neue Auto im Grunde passgenau als „mein Auto“, als Auto „meiner“ Träume 
wiedererkannt werden soll, indem es einerseits vertraute ästhetische Präferenzen bedient, andererseits als das Neue über sie hinausweist, so muss ein Buch in seinem Auftritt (Titelformulierung; Coverästhetik, „wording“ und Nutzenversprechen, Ausstattung, Preis) gleichzeitig konventionell, also vertraut, sein, wie es neu sein muss in der sanften Erweiterung der Erwartungen. Damit aus einer Idee und einem Text ein Buch wird, das in hinreichender Zahl seine Käufer und Leser findet, bedarf es also vielfältiger Überlegungen und einer fortgeschrittenen Kenntnis der Zielgruppe und des Zielgruppenmilieus, in dem das Buch seine Wirkung entfalten soll.

Besonders schwer haben es Buchleute, die im Bereich Belletristik publizieren. Der Konsument hat angesichts der unendlichen Fülle von Romancovern, die ihm in den Auslagen der Buchhandlungen entgegenspringen, den Eindruck, hier gäbe es keine Gesetze, nach denen Bücher auf bestimmte Zielgruppenwelten und Milieus hin designt werden. Vielleicht denkt er, hier müsse sich jedes Buch den individuellen Leser suchen. Und doch täuscht sich der Beobachter. Natürlich sind die Belletristik-Welten aus der Sicht der Macher sortiert. Dafür zwei Beispiele:

1. Taschenbuch-Verleger produzieren nicht ohne Erfolg sogenannte Nackenbeißer; das sind Low-level-Liebes- und Schicksalsromane, die sich durch Cover auszeichnen, die in geringer Variationsbreite das immergleiche Motiv bieten: Von hinten neigt sich ein viriler Gentleman über die Schulter einer freizügig dekolletierten, langhaarigen jungen Dame, die ihm ihre nackte Schulter darbietet, während sie gleichzeitig sinnlich ihre Lippen schürzt. Genauso muss das Cover aussehen, um zum x-ten Mal anzukommen. Die Verleger dieser Bücher wissen mittlerweile ziemlich präzise, dass sie sich mit hohen Limitationen im Sinusmilieu A23 (Traditionsverwurzelte) bewegen, dass sie mit wenigen Ausschlägen in den Bereich BC3 (Hedonisten) rechnen können, in dem sich eigentlich kaum Buchleser befinden, und dass sie insgesamt etwa 10 Prozent am Kuchen des Marktes abgreifen können, während sie bei etwa 90 Prozent des übrigen Buchmarktes heftige Abstoßungsreaktionen hervorrufen.

2. Belletristik-Verleger wissen ziemlich genau, dass sich anspruchsvollere Literatur in einem recht engen Korridor ereignet, der sich von den Konservativen (Sinusmilieu A12), über die Postmateriellen (Sinusmilieu B12) bis in den Bereich der Modernen Performer (Sinus C12) erstreckt. Je anspruchsvoller die Literatur, desto reduzierter, raffinierter, ironischer muss die Ästhetik sein. All das, was in anderen Milieus zieht klare Grundfarben, gefällige, schöne Darstellungen, unverrätseltes "wording" - verhindert hier die Akzeptanz beim gedachten Kunden.

Weitaus einfacher noch ist das Arbeiten mit Sinus-Milieus in anderen Buchsparten. Genannt seien hier die Bereiche Ratgeber und Geschenk- 
buch, bei denen die verlegerische Arbeit ohne Verortung in den Grund orientierungen der Sinusmilieus kaum mehr denkbar erscheint. Käufer von Ratgeber-Büchern - seien es solche zu Hobby, Freizeit, Kochen, Gesundheit oder Wellness - sind in aller Regel Frauen. Sie kaufen Bücher, weil sie „Reduktion von Komplexität“ haben wollen und im Ratgeber ein klares Nutzen-, wenn nicht Glücksversprechen suchen. Der RatgeberVerleger kann diesem Kundenwunsch nur dann entsprechen, wenn er die Erwartungshaltung seiner Leserin sinnlich trifft. Hier muss er sich entscheiden: Will er die erfolgreiche, häufig alleinstehende Businessfrau aus dem urbanen Milieu ansprechen, oder denkt er an Frauen, die "Lisa“ lesen und „Meine Familie \& ich“? Die leben vornehmlich auf dem Land, interessieren sich für andere Themen und haben völlig verschiedene Wertpräferenzen zu ihren Altersgenossinnen aus dem Businessmilieu. Vor allem aber leben sie in einer anderen ästhetischen Welt. RatgeberVerleger haben längst gelernt, dass man sich entscheiden muss, welchen Typ Frau man erreichen will. Natürlich wollen sie beide Typen erreichen, - aber dazu bedarf es unterschiedlich konzipierter Bücher.

\section{Praxis im Verlag: learning by doing}

Um verlegerische Arbeit mit Sinus-Milieus am Beispiel Geschenkbuch konkret zu machen: Der Einstieg geschah in unserem Haus vor Jahren durch ein Beratungsunternehmen, das zur Abwechslung einmal nicht engagiert wurde, weil der negative Gang der Geschäftsdinge es erforderlich machte - im Gegenteil: Wir wollten wissen, warum wir in diesem Bereich offenkundig etwas richtig machten. Mit anderen Worten: Wir wollten unseren Erfolg in seinen Gesetzen verstehen lernen, um aus den Prinzipien neue Erfolge zu generieren.

Unser Berater, der mit dem Sinus-Modell im Konsumgüterbereich exzellente Erfahrungen gemacht hatte, erläuterte die einzelnen Milieus, wie sie statistisch erhoben worden waren. Pro Milieu lernten wir, welche Werte hier typischerweise priorisiert werden, ob das Milieu mehr im städtischen oder ländlichen Bereich angesiedelt ist, wie die Einkommensstruktur ist, welche Medien genutzt werden (und welche in bestimmten Milieus geradezu inexistent sind), welche Kleider getragen, welche Farben bevorzugt, welche Wohnungen bewohnt, welche Wohnungseinrichtungen geliebt, welche Orte besucht, welche Autos gefahren und welche Träume hier geträumt werden. Von typischen Zeitschriften über Alltagsgegenstände, Farbskalen bis hin zu Verpackungen und Geschenkpapieren hatten wir eine Fülle kreativer Elemente zur Hand und bekamen nun die Aufgabe gestellt, daraus sogenannte Moodboards zu 
erstellen, d.h.: Wir sollten Milieus einmal sinnlich anschaubar machen, indem wir die grafischen Elemente den passenden Milieus zuordneten und auf große Pappen aufklebten.

Unter den Teilnehmern der Gruppenarbeit kam es bald zu hitzigen Diskussionen, ob eine bestimmte in Chrom- und Platintönen gehaltene Anzeige tatsächlich vom Milieu Bürgerliche Mitte akzeptiert würde oder nicht. Bald entwickelte sich bei allen Teilnehmern eine intuitive Sicherheit in der Zuordnung von Elementen - und siehe da: Wir hatten schließlich eine Fülle ganzheitlicher Darstellungen, die auf einen Blick die Lebenswelten voneinander abgrenzten. Wir waren überrascht, mit welcher Trennschärfe die Milieus einander gegenüberstanden. Was in einem bestimmten Milieu absolut angesagt ist, wird im benachbarten Milieu als unerträglich empfunden. Da benachbarte Milieus häufig auch überwundene soziale Stufen bzw. Bildungsstufen markieren, sind Abgrenzungen vielfach rigoros. Sozialer Aufstieg setzt sich ästhetisch vom überwundenen Milieu dezidiert $\mathrm{ab}$.

Nachdem wir nun diese Moodboards erstellt hatten, wurden wir aufgefordert, zunächst unsere Bestseller zuzuordnen. Die Erkenntnisse waren verblüffend und vielfältig: 1 . Die erfolgreichen Bücher waren eindeutig bestimmten Milieus zuzuordnen. 2. Es ergab sich ein Kernmilieu für Geschenkbücher, die Bürgerliche Mitte (B 2). 3. Ansonsten waren wir nur noch in zwei anderen Milieus erfolgreich vertreten. 4. Die erfolgreichen Bücher entsprachen in den Farben, der Wortwahl, den Wertpräferenzen absolut dem beschriebenen Milieu. 5. Die Flops (die wir in einem zweiten Durchgang analysierten) waren in jedem Fall durch Unstimmigkeit gekennzeichnet. Uns fiel es wie Schuppen von den Augen: Entweder hatten wir eine Ästhetik gewählt, die nicht zum Milieu passte (wir hatten Ton-in-Ton gearbeitet, wo der Leser klare Grundfarben liebt), oder wir wollten witzig sein und ahnten nicht, dass das Mittel der Ironie in einem bestimmten Milieu nicht verstanden wird. Oder wir wollten in Bereichen reüssieren, in denen Geschenkbücher überhaupt nicht rezipiert werden usw. 6. Die Milieus, in denen es funktionierte, ließen Rückschlüsse auf andere Bücher im Rahmen der Erwartungshaltung zu. 7. Da Milieus sich tendenziell nach oben (zum nächst höheren Milieu) orientieren, konnten wir sehen, wohin wir uns ästhetisch in den nächsten Jahren ausrichten müssen. 8. Da wir Fotos, Anzeigen, Zeitschriften, Designs „unserer“ Milieus vor Augen hatten, konnten wir medienübergreifend lernen, wie es die anderen machen. 


\section{Gibt es einen Transfer in Hinsicht auf Religion?}

In der Kirche gibt es nach wie vor eine heftige Aversion gegen Marketing - deren Teil ja die Arbeit mit Sinusmilieus ist. Diese Aversion ist berechtigt, wenn man darunter eine Haltung versteht, die das Problem der Glaubensvermittlung im Wesentlichen als ein Problem marktorientierter Verpackung ansieht. Marketing wird dann synonym gesetzt mit: die Botschaft des Glaubens mit den anrüchigen Mitteln der Werbebranche verkaufen. Wäre das richtig, dann bestünde das Problem des Nichtankommens der christlichen Botschaft in der mangelnden Präsenz auf Plakatwänden oder im wenig telekonformen Auftritt von Bischöfen. In der Tat ist das Thema Evangelisation nicht durch bischöfliche Imageberatung und die Zuweisung eines ordentlichen Marketingetats an Springer \& Jacoby (eine prominente Werbeagentur) zu lösen.

Evangelisation kann überhaupt nicht delegiert werden, weder an Bischöfe noch an Werbeagenturen; sie ist ein personaler, ganzheitlicher Vorgang, der alle Christen angeht. Dennoch ist es töricht von der Kirche, ihre Botschaft undifferenziert auszurichten - ohne Kenntnis der Lebenswelten, in denen sich die Menschen, an die sich Jesu universaler Heilsauftrag wendet, bewegen. Diese Lebenswelten zu kennen, sie zu beschreiben, sie in ihren Chancen für die Evangelisation zu bedenken, dazu hilft die Marketingarbeit mit Sinus-Milieus. Es genügt eben nicht, die Menschen in Alt und Jung, Christen und Nichtchristen zu sortieren. Unsere Zeitgenossen bewegen sich in parallelen Welten und Milieus, die sich vielfach nur tangential berühren.

Eine Aussage wie: „Wir haben doch die Kirchenzeitung!“ wird beispielsweise wenig helfen, wenn man den Evangelisationsauftrag auch für das Milieu Moderne Performer ernst nimmt. Das ist eine junge, sich betont unkonventionell gebende Leistungselite, in der Multioptionalität und Flexibilität zählen. Hier herrscht ein starker Leistungswille; viele Selbstständige finden sich darunter. Die Leute sind erfolgsorientiert, erproben sich gerne. Sie haben einen hohen Bildungsabschluss und nutzen intensiv die modernen Kommunikationsmittel. Wer nicht weiß, welche Nöte und Sehnsüchte sie bewegen, welche Medien sie nutzen, welche Ästhetik sie anspricht, wird niemals den Anknüpfungspunkt zu ihrer Welt finden. Denn natürlich haben auch diese Menschen einen (mindestens potentiellen) Zugang zum Glauben. Möglicherweise ist ein Newsletter, der beim Thema Reduktion von Komplexität verspricht und ihnen spirituelle Impulse vermittelt, das Medium der Wahl?

Oder was ist mit den vielen Jugendlichen, die auf dem Kölner Weltjugendtag waren und begeistert den Papst feierten? Kennen wir sie wirklich? Wissen wir, wie sie denken, welche Medien sie bevorzugen, welche 
Musik sie hören, was sie lesen (ob sie überhaupt lesen)? Was sind das für junge Leute? Sind sie die Kinder der Bürgerlichen Mitte, die nahtlos eine bestimmte Tradition fortsetzen und wie ihre Eltern angespprochen werden wollen? Oder sind es vielleicht gar die Kinder der Postmateriellen, die Kinder der Altachtundsechziger, die im Protest gegen den Säkularismus der Elterngeneration wieder Interesse am Glauben haben? Soviel weiß man: Sie bewegen sich permanent im Internet, chatten und nutzen ICQ. Könnte man ihnen nicht helfen, Communities zu bilden? „Der Papst“, sagte ein fassungsloser Beobachter am Rand der Kölner Geschehnisse, „müsste denen vier mal im Jahr eine E-Mail schicken! Die würden sogar seine Enzykliken lesen!“

Die aktuelle Sinus-Studie wird manchen katholischen Büchermacher enttäuschen, der breitere lebensweltliche Übereinkünfte mit der katholischen Kirche von ihr erwartet hat. Es gibt nur wenige Milieus, in denen überhaupt Bücher gelesen werden, und die Auskünfte dort über Kirchenbindung und Erwartungen an die Kirche sind ernüchternd. Gleichwohl ergeben sich bei näherem Hinsehen Chancen.

\section{Chancen für katholische Büchermacher: learning by Pater Anselm}

Unglücklicherweise sind die der Kirche nahe stehenden Milieus (eine parallel durchgeführte Untersuchung des deutschen Buchhandels fand das heraus), die Sinus-Milieus der Traditionsverwurzelten, eher keine Buchkäufer. Nun sind diese ca. 14 Prozent der Gesamtgesellschaft auch die eher Älteren.

Eine kirchliche Kommunikationsstrategie über Bücher dürfte also wenig zielführend sein, wenn man mehr erreichen will, als sich lediglich der Affirmation der kirchentreuen Klientel zu versichern. Nüchtern betrachtet heißt das für katholische Büchermacher, dass ein Programm nur Chancen hat, das sich an den religiösen Bedürfnissen und Erwartungen der eher jüngeren und gebildeteren Milieus ausrichtet. Diese Milieus heißen bei Sinus Etablierte, Bürgerliche Mitte, Postmaterielle und Moderne Performer. Die Studie gibt darüber hinreichend Auskunft. Es ist zwingend zu wissen, wie diese Milieus „ticken“, was ihnen Kirche und Religion bedeutet und welche Erwartungen sie an die Kirche stellen. Wie man sie erreicht, was der Buchmacher beachten muss, kann er oben bei den Ausführungen zum Geschenkbuch nachvollziehen.

Die katholischen Büchermacher finden in diesen Milieus ihre Kernzielgruppen. Aus diesen Leser-Milieus erwachsen aber auch die potenziell engagierten Laien, auf die eine unter Priestermangel leidende Kirche 
in Zukunft mehr und mehr angewiesen ist. Der Kirche eröffnet sich im Zugang auf diese häufig kirchenkritischen und kirchenfernen Milieus die Chance, sich ihnen auf neuartige Weise zeitgemäß und attraktiv zu präsentieren. Dass das grundsätzlich möglich ist und ansatzweise bereits schon geschieht, beweist bereits höchst erfolgreich der Bestsellerautor und Benediktiner Anselm Grün. 